\title{
Research on the Effect of Substation Main Wiring on the Power System Reliability
}

\author{
Yuxin Zhao ${ }^{1, \text { a }}$, Tao Liü ${ }^{2, b}$ and Yanxin Shi ${ }^{3, \mathrm{C}}$ \\ ${ }^{1}$ Tianjin Electric Power Corporation Electrical Power Research Institute, Tianjin ,China \\ ${ }^{2}$ Tianjin Electric Power Corporation Electrical Power Research Institute, Tianjin ,China \\ ${ }^{3}$ Tianjin Electric Power Corporation Electrical Power Research Institute, Tianjin ,China \\ aemail:yx_zhao1101@163.com, ${ }^{\text {bemail:net_liutao@126.com, }{ }^{\mathrm{c} e m a i l}: y a n x i n s h i 786 @ y a h o o . c o m . c n}$
}

Keywords: reliability; substation main wiring; power system; the analytical method; the Monte Carlo simulation method

\begin{abstract}
The research of the reliability will be further into the substation level with the combination of the substation main wring and power system. In this paper, the power system reliability considering the effect of the substation main wiring reliability evaluates by two different methods which are the analytical method and the Monte Carlo simulation method. The substation main wiring is analyzed and combined into the reliability model in order to more accurately calculate the reliability of the system. In this paper, it proves the necessity of considering the effect of substation main wiring on the power system reliability by the calculation of the IEEE RTS example systems.
\end{abstract}

\section{Introduction}

The reliability of the power system is to measure the power system with acceptable quality standards and required number of uninterrupted power supply to users of electricity[1]. It is the guiding power system planning, operation, construction and management science[2]. It is important to ensure safe and reliable power supply. Grid collapse and a large area power outage will not only cause enormous economic losses, affecting the normal life of the people, but also endanger public safety, causing serious social influence[3].The frequent occurrence of worldwide blackouts in recent years[4,5], make people aware of the importance of the reliability of power system more deeply.

As an important part of power system, substation is the middle link of power plants and users, and plays the role of transformation and distribution of electricity power. The safe operation of the substation has an important effect on the development of the whole power supply reliability, and even the entire national economy and the people's living standard. So the reliability level of the main wiring has a great influence on power system reliability. In order to analyse the power system reliability, it is necessary to consider the effect of substation main wiring reliability.

At present, the reliability research mainly focus on the adequacy of the transmission system. The research of the substation main wiring reliability has gradually been expanded, and the association of the substation safety and reliability of transmission system still needs to be further expanded.

\section{Substation Main Wiring Reliability Model}

The reliability model of transmission line, transformer and switch. Transmission line transformer can be in one of the following states, which are normal operation state, repairing state with fault and planned maintenance state. The state transition model can be used is shown in Fig. 1.

In the figure1, State $\mathrm{N}$ represents the normal operation state. $\mathrm{M}$ represents planned maintenance state. $\mathrm{R}$ represents repairing state with fault. ${ }^{\lambda_{M}}$ represents planned maintenance rate. ${ }^{\lambda}{ }$ represents rate of repairing state with fault. ${ }^{\mu}$ represents planned maintenance repair rate. ${ }^{\mu_{R}}$ represents the maintenance rate of the repairing state with fault. 


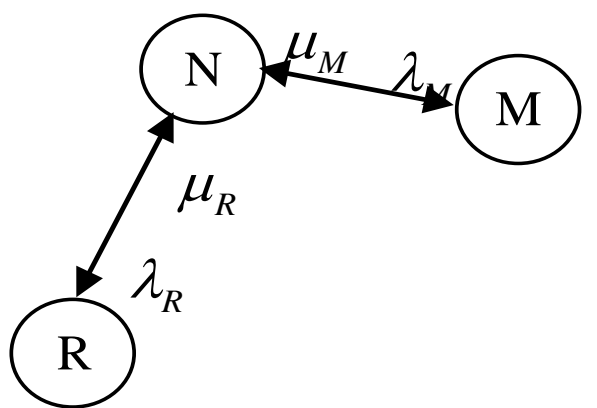

Fig. 1 the reliability model of transmission line

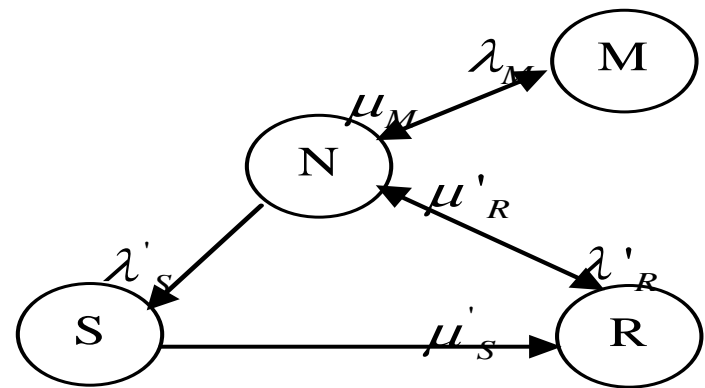

Fig. 2 the reliability model of breaker The reliability model of breaker. A normally closed breaker (Fig. 2) general may have the following 7 State. $\mathrm{N}$ represents the normal operation state. $\mathrm{M}$ represents planned maintenance state, and $\mathrm{m}$ represents the force repairing state. $\mathrm{F}$ the maloperation state, I represents the ground or insulation fault state, st represents the refusing action state, $\mathrm{R}$ represents the state of after fault restoration. The states of the breaker are merged, in order to simplify the breaker model. The maintenance states should not be combined as it is considered that the planned maintenance is not a random occurrence, but man-made plan deterministic state is.

The reliability model of Relay protection. Relay protection is reflected through the breaker. Breaker executes the protection command, and the protection acts on the system by the action of circuit breaker. The consequences of the Relay protection maloperation make the same effect with the protected element with the S type fault. So the maloperation of S type failure rate can be added to the breaker in the rate of protective relay device. The new state is called the $\mathrm{F}$ type fault state. The reliability model of the breaker considering the effect with the relay protection fault is shown in Fig. 3.

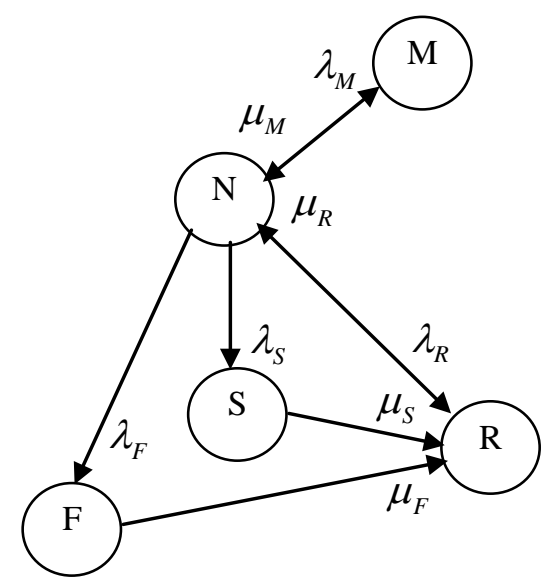

Fig 3 the reliability model of the breaker considering the effect with the relay protection fault

The reliability model of bus. The reliability model of the bus without switching operation is similar with the model of the transmission line, transformer and generator shown in Fig. 1.

The reliability model of the bus with switching operation is similar with the model shown in Fig. 2, only the significance of rates $\lambda_{s}$ and $\lambda_{R}$ is a little different, which is not considered the factors of refusing action and maloperation. $\mu_{s}$ represents the switching rate, only the reciprocal of the switching time.

\section{Evaluation Method}

In this paper, the effect of substation main wiring on the reliability of the system is evaluated by two methods.

The reliability evaluation with the Monte Carlo simulation method. Considering the characteristics of each component above mentioned, the element sampled with the Monte Carlo simulation method, can be unified described as Eq. 1, Eq. 2 and Eq. 3. 


$$
0<x \leq P L_{i}
$$

$P L_{i}<x \leq P L_{i}+P S_{i}$

$P L_{i}+P S_{i}+P M_{i}<x \leq P L_{i}+P S_{i}+P M_{i}+P R_{i}$,

In the equations, $P L_{i}$ represents the probability of relay protection in the refusing action, ${ }^{P S_{i}}$ represents the probability of the element in short circuit fault, ${ }^{P M_{i}}$ represents the probability of the element in maintenance the state. ${ }^{P R_{i}}$ represents the probability of the element in fault.

All elements of the substation are sampled to simulate the fault state with the Monte Carlo simulation method. If there is a element sampled in some fault, it is analysed whether causes line outages. If there is, system elements will be regarded as the fault elements.

The reliability evaluation with the analytical method. The and-order and below 3nd-order fault states are enumerated to analyse the main wiring reliability with the analytical method. The single fault states Include R state, M state, S state and RL state mentioned above. The fault states to be enumerated are the state of the single faults and the combinations of single faults.

The minimal path and element path matrix of graph theory methods are adopted to the main wiring reliability analysis[6]. No longer detailed here.

With the probabilities of all fault combinations, it can be calculated the outage probability of all the lines. And the probability of the corresponding the element in the system can corrected with the Eq. 4.

$$
\mathrm{FOR}_{j}=\mathrm{FOR}_{j}+P_{i}-\mathrm{FOR}_{j} \times P_{i}
$$

\section{The reliability evaluation of the system}

The reliability model of the system. In this paper, it only considers that the generator and the transmission line only can be in operation state and fault states, and the probabilities have two-point distribution.

The states of all the elements in the system form the state vector of the $\mathrm{x}$. All possible states of $\mathrm{x}$ in the set $\mathrm{X}$ is called the state space system.

System connectivity analysis.By the way of the detection if the singularity of the Jacobian Matrix, it can be judged the system islanding. The numbers of will be recorded, and then the next detection continues. If the network is not split into system islanding, the evaluation of the system state will continue.

Power flow calculation. Making a contingency analyze is involving DC power flow calculation, Depth-first Search for recognize of system islanding, and load curtailment calculation. In this paper, DC power flow calculation is adopted which can meet the requirements of the accuracy with smaller computational complexity and faster calculation speed. At the same time, DC power flow calculation provides convenience for the optimization algorithm

The DC power flow calculation model can be described as the Eq. 5

$$
P_{g}-P_{d}=B \theta
$$

In this equation, ${ }^{P_{g}}$ presents the generator output vector, ${ }_{d}$ presents load vector, $\theta$ presents the node voltage phase angle vector, $B$ presents the node admittance matrix.

The line active power is described as the Eq. 6

$$
P_{i j}=\frac{\theta_{i}-\theta_{j}}{x_{i j}}
$$

In this equation, ${ }^{{ }_{i j}}$ presents the node active power, ${ }^{X_{i j}}$ presents the branch reactance, $\mathrm{i}$ and $\mathrm{j}$ present the two ends of transmission line. 
Overload correction. In this paper, the linear programming is used to calculate the minimum curtailment of the load quantity. So the LINDO programming developed by Chicago University is adopted to improve calculation efficiency significantly.

\section{Algorithm Explanation}

In this paper, the analytical method and Monte Carlo simulation method are used to evaluate the effect of main wiring on the power system reliability.

The algorithm is explained by the following steps.

Step 1: Read system and substation parameters and their correspondence data.

Step 2: Set the maximum sampling number, use the system time as a random number seed initial, and then begin sampling.

Step 3: For the analytical method, enumerate the and-order and below 3nd-order fault states, analyse each fault state whether it causes the line outage. If it is, record the line outage probability, and go to Step 4. If not, go to Step 5. For the Monte Carlo simulation method, sample the elements of the substation according to the Eq. 1, Eq. 2 and Eq. 3, and then analyse each fault state whether it causes the line outage. If it is, regard the generator and transmission line of the system corresponding to the line with outage as a fault, and the go to Step 5. If not, go directly to Step 5.

Step 4: Correct the line outage probability to the corresponding generators or the transmission lines of the system according to the Eq. 4

Step 5: Sample the elements of the system, judge and evaluate the connectivity of the system in the sampling state. Then calculate the power flow according Eq. 5 and Eq. 6 and use LINDO programming for overload correction.

Step 6: Judge the sampling number whether it is bigger than the maximum sampling number. If it is, go to Step 7. If not, go to Step 4 for the analytical method, and go to step 3 for the Monte Carlo simulation method.

Step 7: Calculate the reliability index including LOLP,EENS, and etc..

\section{Analysis of example}

The IEEE RTS example system reliability is evaluated by the methods mentioned above. The system wiring is described in reference 7 and the IEEE RTS substation main wiring is described in reference 8 . The maximum sampling number is 100000 .

Comparison of comprehensive index. The calculation results are shown in Table 1.

Such as LOLP, EENS and the number of system islandins shown in Table 1, the reliability indexes considering the effect of the main wiring are much higher than those not considering the effect of the main wiring. It proves that the effect of main wiring is necessary to the system reliability evaluation. Further more, the indexes are higher by the Monte Carlo simulation method than the analytical method. It is because that the Monte Carlo simulation method can detect the high-order fault states and the common mode faults which are regarded as the cause of the blackout. The indexes are closer to the real by the Monte Carlo simulation method.

Table1 Comparison of comprehensive index

\begin{tabular}{cccc}
\hline \multirow{2}{*}{ index } & Without main wiring & \multicolumn{2}{c}{ With main wiring } \\
\cline { 3 - 4 } & & the analytical method & the Monte Carlo method \\
\hline LOLP & 0.0857 & 0.0914 & 0.1082 \\
EENS[MWh] & 130420. & 141037 & 67055 \\
System Islanding & 30 & 413 & 1529 \\
\hline
\end{tabular}

The curtailment of the load quantity. The curtailment of the load quantity is shown in Table 2. The unit is MWh.

Like the reliability indexes, the curtailment of the load quantity considering the effect of the main wiring is also more higher than those not considering the effect of the main wiring, and with only some exceptions, it is also higher by the Monte Carlo simulation method than the analytical method 
especially from node 3 to node 10 . Take the node 9 and node 10 for example. the main wiring of the node 9 and node corresponding is angle scheme, which use a breaker connecting the two angles. If the breaker is in fault, the whole substation will be in outage. The transmission lines of the system corresponding to the nodes will be in outage, too. So the curtailment of the load quantity will greatly increase.

Above all, the effect of the substation main wiring on the system reliability is more significant by the Monte Carlo simulation method.

Table2 The curtailment of the load quantity

\begin{tabular}{cccc}
\hline \multirow{2}{*}{ node } & \multirow{2}{*}{ Without main wiring } & \multicolumn{2}{c}{ With main wiring } \\
\cline { 3 - 4 } & 4930.123 & the analytical method & the Monte Carlo method \\
\hline 1 & 4427.981 & 5179.281 & 5614.537 \\
2 & 8217.310 & 4651.762 & 5042.686 \\
3 & 3378.047 & 8632.573 & 9357.562 \\
4 & 3241.099 & 3573.625 & 5136.995 \\
5 & 6208.303 & 3427.830 & 6298.962 \\
6 & 6025.451 & 6557.798 & 8440.222 \\
7 & 7806.028 & 10275.673 & 9892.807 \\
8 & 7988.625 & 8197.619 & 13533.360 \\
9 & 8901.611 & 8392.353 & 10829.920 \\
10 & 12097.061 & 9351.479 & 13993.073 \\
13 & 8855.962 & 12708.421 & 13776.411 \\
14 & 14470.824 & 9303.523 & 10085.372 \\
15 & 4564.929 & 15202.149 & 16479.707 \\
16 & 15201.212 & 4795.630 & 5198.646 \\
18 & 8262.521 & 15969.450 & 17311.490 \\
19 & 5843.109 & 8680.091 & 9409.549 \\
20 & & 6138.407 & 6654.266 \\
\hline
\end{tabular}

\section{Conclusion}

In this paper, the effect of substation main wiring on the power system reliability is considered which is more precise to evaluate the power system reliability. Two methods are used to evaluate the effect of substation main wiring on the power system. The results of the analysis of example prove the necessity of considering the effect of the substation main wiring on the system reliability, and the Monte Carlo simulation method.is more significant.

The main achievement also can be used to evaluate the main wiring reliability or to evaluate effect of different substation main wiring on the system. So it also can provide the basis for the choice for the main wiring.

\section{References}

[1] Yongji Guo, Analysis of the Power System Reliability, Tsinghua University Press,2003.

[2] Yongji Guo, Strengthen the research and Application of thee power system reliability-Thinking of eastern North America blackou.Automation of electric power systems Journal ,2003,27(19).

[3] Xizheng Zhao, Strengthen the safety, security and reliability of power system,Grid Technology Journal,2003,27(10).

[4] Deqiang Gan, Hu Jiangyi,Han Zhenxiang,Thinking on several blackouts in the world in 2003,Automation of electric power systems Journal ,2004,28(3).

[5] Baosheng Tang, The blackout area of London and its lessons, Grid Technology Journal, 2003,27(11). 
[6] Xifan Wang, Research on the reliability of power station main wiring(1)-The Basic model and algorithm, Xi’ an Jiao Tong University Journal,1990,23.

[7] IEEE Committee Report. IEEE Reliability Test System. IEEE Transactions on Power Apparatus and System,1979,98(6)

[8] The IEEE Reliability Test System - 1996, IEEE Transaction on Power System,1999,14(3). 